\title{
ANTOLOGÍAS DE TEXTOS TRADUCTOLÓGICOS: VÍAS DE RECEPCIÓN MUTUA DE TEMAS EN EL ÁMBITO CHECO - HISPÁNICO ${ }^{1}$
}

Jana KRÁLOVÁ

Universidad Carolina, Praga

\begin{abstract}
En): In recent decades, anthologies have become somewhat of a popular genre within the field of translation studies, but the majority of research has focused on collections of literary translations; analysis devoted to anthologies of theoretical work remains rare. Czech and Spanish research, however, proves to be an exception to this rule. This text examines the general characteristics of critical anthologies as a specific genre resulting from an author or editor's evaluation, selection, and (re)arrangement of appropriate studies. It also outlines the role anthologies have played in the mutual reception of theoretical texts that have led to translation studies being integrated into the broader humanities in both the Czech and Spanish cultural spheres.
\end{abstract}

Key words (En): anthologies; translation studies development; Czech culture; Hispanic culture

Palabras clave (Es): antologías; evolución de la traductología; recepción; cultura checa; cultura hispánica

\section{A modo de introducción}

Las antologías representan un género relativamente nuevo en los estudios sobre la traducción, ya que su reflexión teórica no aparece sino en 2010 (SERUYA et al.), libro que pone énfasis en las antologías de textos traducidos, siendo las dedicadas a la reflexión teórica relegadas al segundo plano (SABIO PINILLA, 2011: 160). Otro aspecto que despierta la atención es el hecho de que este tema aparezca con relativa frecuencia entre los teóricos españoles (SABIO PINILLA, 2010) y, más recientemente, entre los traductólogos los checos ${ }^{2}$. El objetivo del presente artículo es ofrecer un esbozo del papel que desempeñan las antologías de textos traductológicos como géneros de la metatraductología histórica en los intercambios culturales y en la evolución de la disciplina, poniendo énfasis en el área checo-hispánica.

\section{Característica general de la antología como género}

La antología como género es considerada como resultado de los procesos de selección de los respectivos textos, su evaluación y reconfiguración conforme a la concepción del antólogo como autor secundario, que obedece, generalmente, a los siguientes motivos: intención de transmitir un mensaje particular (moral, religioso, sentimental, ideológico); ilustración de algún tema específico (por ejemplo, con motivo de un aniversario); presentación de un específico modo de expresión o

\footnotetext{
${ }^{1}$ La publicación del texto forma parte del proyecto de investigación de la Universidad Carolina Progres Q10 Jazyk v proměnách času, místa, kultury.

${ }^{2}$ Las reflexiones que presentaremos a continuación se basan ante todo en la reedición de la obra LEVÝ (1957) y en las experiencias didácticas con la preparación de las antologías de estudios teóricos de los investigadores españoles y latinoamericanos (VAVROUŠOVÁ, 2013; 2015). 
corriente artística; posibilidad de compartir los textos preferidos por el antólogo con los lectores (SERUYA et al., 2010: 4). Asimismo, entre otros factores que influyen en la confección de las antologías pueden citarse los siguientes: la figura del compilador, los derechos del autor, los factores económicos, la política de la respectiva editorial y el presunto lector.

En cuanto a los tipos de antologías según los textos que las forman, SABIO PINILLA (2010:11) habla de antologías generales, las cuales pueden subdividirse en las antologías de tipo universal, las que parten de una tradición (p. ej. ámbito checohispánico), en las generales de carácter nacional, y en las parciale, que incluyen un período determinado. En este lugar cabe mencionar un aspecto que tal vez haya pasado desapercibido por los investigadores en el área: el de la evolución de la respectiva disciplina, que influye no solamente en la selección y configuración de los respectivos textos que forman la antología, sino en primer lugar en los peritextos que los acompañan, como son el estudio inicial, las notas y enmiendas del compilador, los cuales no deben superar el $25 \%$ del total (SABIO PINILLA, 2011: 162), dato cuya importancia se demostrará más adelante.

\subsection{Antología de textos teóricos como género en el ámbito hispanohablante.}

SABIO PINILla (2010) relaciona la proliferación de las antologías en el área hispánica (y lusófona) en los años noventa del siglo XX con la fundación y desarrollo de las Escuelas, Facultades o Departamentos de Traducción e Interpretación en varias universidades españolas por una parte, y, por la otra, con la intención de dotar a la disciplina de una perspectiva histórica. Por lo tanto, no sorprende el hecho de que varios de los antólogos españoles estén relacionados con la(s) universidad(es) y que la preparación sirva por una parte para cubrir un vacío del conocimiento, y por la otra, para dotar a la disciplina de una perspectiva histórica. De allí deriva que, en el ámbito hispánico y lusófono, «las antologías sobre la traducción son un tipo de obra histórica que proporciona un conjunto de textos de la tradición occidental y de otras tradiciones menos conocidas con una finalidad fundamentalmente didáctica e investigadora» (SABIO PINILLA, 2011: 165).

\subsection{Antología de textos teóricos como género en la traductología checa}

La traductología checa tiene cierto privilegio en el estudio de los conjuntos de textos traductológicos: el de disponer de la primera obra en esta área, dedicada al estudio de la traducción en una literatura nacional (la checa) acompañada de una extensa selección de textos originales dedicados al estudio de la traducción, publicada ya en 1957 (LEVÝ, 1957), es decir, casi cuarenta años antes del auge del género a escala internacional. Cabe destacar también otra característica: la obra antológica se sale del marco de la antología tradicional, ya que los peritextos rebasan el $25 \%$ del total de volumen, atributo mencionado por ejemplo por SABIO PINILLA (2011: 162).

Al tratarse de un libro modélico, cuya importancia no se ha superado hasta ahora, no sorprende que el género de colecciones de textos traductológicos siga vivo hasta la actualidad a pesar del tiempo transcurrido; así, por ejemplo, en el área de 
las relaciones con el mundo hispanohablante y lusoparlante, en las últimas décadas destacan las publicaciones de VAVROUŠOVÁ (2013; 2015), cuyo objetivo es precisamente el de «cubrir el vacío de conocimiento» sobre la investigación en las mencionadas áreas lingüísticas y culturales.

\subsection{Antologías de textos teóricos y el dinamismo de la traductología}

Como advierte SABIO PINILla (2010: 12), «la antología es un procedimiento que permite reformular el pasado desde el presente». De allí se desprende otro problema: ¿cuál es el presente de la antología? Al tratarse de una selección y recontextualización de textos, el antólogo como autor secundario no puede prescindir de las normas de recepción de su época, fenómeno que se refleja no solamente en los textos que la conforman, sino también en los peritextos que acompañan los textos seleccionados. Por lo tanto no es de sorprender que las antologías envejezcan a otro ritmo que los textos que las forman, especialmente si el presente de una antología publicada hace décadas ya se ha convertido en historia.

Es lógico, pues, que la renovación metodológica de la traductología de las últimas décadas del siglo XX y de las primeras del XXI (LAMBERT, 2010: 40)-que tiene como efecto cierta reconfiguración de los textos que forman el canon de la disciplina y de su nueva interpretación-, sin duda influya no solamente en la preparación de nuevas antologías de textos traductológicos, sino también en la valoración de las escritas ya hace décadas. Como ejemplo puede citarse la valoración del libro de LEVÝ (1957) por parte de ULIČNÝ (2016), quien relaciona la relativamente escasa atención dedicada por Jiř́i LEVÝ (1957) a las traducciones del español con la evolución de los estudios hispánicos en el ámbito checo:

«Al evaluar la importancia atribuida por Jiří Levý a los problemas de las traducciones checas del español hay que tener presente que la literatura española (e hispanoamericana) se encontraba durante mucho tiempo al margen del interés no solo por parte de los traductores, sino también de los historiadores y críticos de las literaturas universales, hasta casi los años noventa del signo XIX, cuando aparece la figura del hispanista Antonín Pikhart. Es que los países de la Corona Checa carecían de conocedores del español y, por consiguiente, las traducciones eran escasas y, además, "de segunda mano"». ${ }^{4}$

Asimismo, el dinamismo de la disciplina de la Traducción representa otro impulso para la preparación y publicación de las antologías recientes

\footnotetext{
${ }^{3}$ Esto no quiere decir que la literatura española carezca de interés en la obra de Levý, cuyos estudios versológicos no solamente incluyen el verso romance y español; asimismo, fue él quien impulsó la traducción del Libro de Buen amor al checo (RuBÁš, 2011: 89; KRÁlOvÁ, 2018: 100). Por otra parte, se pueden mencionar textos escritos por otros autores dedicados a las traducciones del español, publicados en el mismo período: por ejemplo, en 1957, la revista Časopis pro moderní filologii publicó el artículo de Eduard Hodoušek, dedicado al escritor checo Josef Kajetán Tyl como primer traductor de Calderón (HoDOUŠEK, 1957)

${ }^{4}$ «Při hodnocení vhledu Jiřího Levého do problematiky českých překladů ze španělštiny je třeba si uvědomit, že španělská (a hispanoamerická) literatura stála dlouho stranou zájmu českých překladatelů, ale též historiků a kritiků světových literaturu nás, a to téměř až do devadesátých let 19. století, kdy se zaníceným hispanistou stává A. Pikhart. Nebylo totiž v zemích Koruny české znalců španělského jazyka, a tudíž se překládalo sporadicky, a navíc "z druhé ruky".»
} 
(VAVROUŠOVÁ, 2013; 2015): además de «llenar un vacío de conocimiento» que se deriva de la relativa escasez del conocimiento del español, en comparación con el inglés, el alemán y el francés entre los especialistas checos, la compilación de los textos refleja también el avance de los estudios traductológicos en el área hispano y lusoparlante. Obviamente, y como consecuencia de lo constatado, al evaluar la concepción de las antologías no se puede prescindir de la influencia que ha venido ejerciendo la intención de renovar la metodología traductológica que se refleja en la atención dedicada a las concepciones, métodos y culturas que carecían de interés hasta hace poco.

\section{Traductología checa en las antologías escritas en español}

Como afirma SANTOYO MEDiAviLla (2011: 1984), la historia de las traducciones en España carece de conocimientos más profundos de las traducciones de las lenguas eslavas, fenómeno que puede extenderse también al conocimiento de los conceptos teóricos. En cuanto a las antología publicadas por autores hispánicos y lusófonos, SABIO PINILLA (2010: 9-10) cita catorce antologías publicadas en España entre 1987 y 2007. A pesar de la variedad de temas, épocas y autores incluidos, la única en la que aparece un texto checo es la de VEGA CERNUDA (1994: 325-327), basado en la versión alemana. En ella se proporciona al lector la traducción española del capítulo sobre la doble norma de la traducción propuesta por LEVÝ (1963). Como admite el compilador (VEGA CERNUDA, 2011: 14; 2013: 16), la repercusión del capítulo en el ámbito hispanohablante fue muy reducida, si no nula.

\subsection{Jiř́i Levý: una concepción (re)descubierta}

En 2013, los investigadores del Instituto de Traductología de la Universidad Carolina de Praga publicaron una recopilación de textos de Jiř́ Levý destinada al público hispanohablante (KRÁlOVÁ, CUENCA 2013). La concepción del libro como antología dedicada a la obra de un autor se justifica por varios motivos, siendo uno de ellos la publicación de la versión inglesa de su obra principal, LEVÝ (1963), un año antes por una parte, y por la otra, la intención de presentar al público internacional, en este caso hispanohablante, los textos de Levý de una forma más compleja. Este ha sido el motivo por el cual el libro incluye una selección de cinco textos, dos de los cuales (el prólogo y el epílogo LEVÝ, 1957) y el estudio Čapkovy překlady ve vývoji českého překladatelství a českého verše [Las traducciones de Karel Čapek en la evolución de la traducción y del verso checos] (LEVÝ 1971), no habían sido presentados al público internacional. La presentación de dichos textos tuvo dos motivos: en el primer caso, los compiladores y traductores sostienen la opinión de que, al tratarse de un trabajo previo a la obra fundamental de LEVÝ (1963), su conocimiento facilita la comprensión de varios aspectos del libro, basados en un profundo conocimiento de la literatura checa; el segundo texto sirve para ilustrar el método de Levý posterior a la publicación de LEVÝ (1963). La obra 
fue reseñada por SERRANO BERTOS (2014), quien pone énfasis en la base metodológica del autor checo, y por VAVROUŠOVÁ $\left(2015^{\mathrm{a}}, 2016\right)$.

El creciente interés por la obra de Jiř́ Levý en el mundo hispanohablante se patentiza también en BOLAÑOS (2016). Aunque no se trata de antología propiamente dicha sino de un libro de textos, el autor colombiano (BOLAÑOS, 2016: 209 - 246) incluye un amplio estudio dedicado a Jiří Levý; entre otras cosas, incorpora la obra de Levý en el contexto actual y lo considera como uno de los fundadores del cognitivismo en los estudios de la traducción.

\section{Traductología hispánica en las antologías checas}

El estudio de la representación de la traductología hispánica en la investigación checa no puede prescindir del análisis de sus vínculos con dos áreas de conocimiento: el hispanismo y la traductología como tal. Como ya se ha señalado (ULIČNÝ 2016), la presencia de las reflexiones sobre las traducciones de la literatura española al checo resultan escasas en comparación con las «grandes» literaturas europeas de la época en la mencionada antología por LEVÝ (1957), fenómeno que se patentiza todavía más en la segunda edición del libro, publicada en $1996 .{ }^{5}$ Además, hay que tener presente que la antología reúne textos escritos por traductores y otros intelectuales checos, concepción que reduce la posibilidad de presentar textos de otras áreas culturales. Por ello sorprende que al menos un comentario de origen español acerca de la traducción fuera conocido entre los intelectuales checos de la segunda mitad del siglo XIX: el del «revés de tapiz» del Quijote, que aparece en el texto de Durdík (LEVÝ, 1957: 427).

Sin embargo, el avance de los estudios hispánicos por una parte y el nivel alcanzado por la traductología en los países hispanohablantes y lusófonos repercutieron en el interés por preparar dos antologías de textos teóricos en el siglo XXI (VAVROUŠOVÁ, 2013; 2015), que se presentan a continuación. ${ }^{6}$

\subsection{Sedm tváŕí translatologie (VAVROUŠOVÁ, 2013)}

La primera antología Sedm tvárí translatologie [Siete caras de la translatología] incluye siete estudios, cuyos autores (Julio César Santoyo Mediavilla ${ }^{7}$, Miguel Ángel Vega Cernuda ${ }^{8}$, Fernando Navarro Domínguez ${ }^{9}$, Antonio Bueno García ${ }^{10}$,

\footnotetext{
${ }^{5}$ El único texto traducido de una lengua románica es la versión checa del ensayo de Germaine de Staël Sulla maniera e la utilità delle traduzioni (LEVÝ, 1957: 324-330)

${ }^{6}$ Más detalladamente, véase KRÁLOVÁ, 2017.

${ }^{7}$ Originales: SANTOYo Mediavilla Julio César (2008), De nuevo sobre El Tostado: La creación de un metalenguaje traductor en la España del siglo XV, in: Historia de la Traducción: Viejos y nuevos apuntes, León, p. 103-117; SANTOYo Mediavilla Julio César (2008), Un quehacer olvidado: Los intérpretes-traductores en navíos, in: Historia de la Traducción: Viejos y nuevos apuntes, León, p. 103117.

${ }^{8}$ Original: Vega CERnUdA Miguel Ángel (2002-2003), Una mirada retrospectiva y escéptica a la teoría de la traducción, Hieronymus Complutensis 9-10, p. 63-76.

${ }_{9}^{9}$ Original: NAVARro Domínguez Fernando (ed.) (2000), Introducción a la teoría a y práctica de la traducción. Ambito hispanofrancés, Alicante.

${ }^{10}$ Original: BuENO GARCíA Antonio (2011), El espíritu religioso y patriótico en la traducción. La obra de fray Vicente Solano, in: Bueno García Antonio, Vega Cernuda Miguel Ángel (ed.) Los franciscanos hispanos por los caminos de la traducción: textos y contextos, Soria p. 695-721.
} 
Manuel Ramiro Valderrama ${ }^{11}$, Raquel Merino Álvarez ${ }^{12}$ pertenecen a la que podría considerarse «generación fundadora» de los estudios de traducción e interpretación en España. En la época de la publicación ya habían presentado los resultados de su investigación como conferenciantes en las universidades checas, ponentes en congresos etc., es decir, no eran desconocidos por el público doméstico (VAVROUŠOVÁ, 2013: 7).

Los textos escogidos resultan no solamente representativos para los autores individuales, sino que se caracterizan también por cierta fuerza inspiradora para los estudios de traducción e interpretación en el contexto doméstico: citemos como ejemplo los estudios de Julio César Santoyo Mediavilla orientados a diferentes aspectos diacrónicos del trabajo de los traductores e intérpretes y su importancia para la actualidad. Clara finalidad basada en una larga trayectoria investigadora y docente se manifiesta en los estudios de Miguel Ángel Vega Cernuda y Fernando Navarro Domínguez. La contribución de Antonio Bueno García ofrece un tema hasta el momento poco estudiado en el ambiente checo: el de la traducción en las órdenes religiosas. Las mismas características, es decir, la introducción de un tema relativamente poco estudiado en la época de la publicación del libro, la censura, aparece en el texto de Raquel Merino Álvarez. La contribución de Manuel Ramiro Valderama presenta un problema específico del ambiente hispánico: el de la variabilidad dialectal del español y su papel en la traducción.

Los textos van acompañados por un estudio introductorio que caracteriza a los autores y los temas presentados y explica la importancia de los estudios incluidos en la antología para subrayar la evolución de la disciplina; asimismo se ofrece una breve información biobibliográfica que introduce los textos traducidos.

\subsection{Překlad a tlumočení jako most mezi kulturami (VAVROUŠOVÁ, 2015)}

La segunda antología Překlad a tlumočeni jako most mezi kulturami [La traducción e interpretación como puente entre las culturas] está dedicada a la traductología iberoamericana e incluye la selección y traducción de textos de Adriana Domínguez Mares (México) ${ }^{13}$, Ricardo Silva-Santisteban (Perú), ${ }^{14}$ Martha

11 Original: RAMIRO VALDERRAma Manuel (2007), La traducción interlectal en lenguas transnacionales: Esbozo de una teoría para el español, in: Alcira García-Vassaux (ed.) Memoria del II Congreso de Interpretación y Traducción en La Antigua de AGIT y I Encuentro Internacional del Centro Regional América Latina de la FIT, Ciudad de Guatemala, p. 147-177.

${ }^{12}$ Original: Merino Álvarez Raquel (2001), Textos dramáticos traducidos y censurados en la España de Franco (años sesenta): el teatro y el cine, espectáculos controlados, in: SANDERSON John D. (ed.): !Doble o nada!: Actas de las I y II Jornadas de doblaje y subtitulación de la Universidad de Alicante, Alicante 2001.

13 Original: Domínguez MAREs Adriana (2008) Doña Marina: primera intérprete mexicana, in : Bravo Utrera Sonia, García López Rosario (eds.), Estudios de Traducción: Problemas y perspectivas. Universidad de Las Palmas de Gran Canaria, pp. 81-98;

${ }^{14}$ Original: Silva-SANTIESTEBAn Ricardo (2011), Garcilaso de la Vega el traductor, in : Entre enigmas y certezas: cómo traducir literatura, Lima, Universidad de Ricardo Palma, p. 49-68 
Pulido Correa (Colombia), ${ }^{15}$ Lourdes Arencibia Rodríguez (Cuba), ${ }^{16}$ Daniel Ricardo Yagolkowski (Argentina), ${ }^{17}$ Iván Pinto Román (Perú) ${ }^{18}$ a Célia Luiza Andrade Prado (Brasil). ${ }^{19}$ Los textos van introducidos por un prólogo que explica la selección de los temas y por un profundo estudio dedicado a la historia y actualidad de los estudios de Traducción en Iberoamérica. Además de ofrecer un esbozo de la evolución de la traducción en América Latina, poniendo énfasis en el papel que ha venido desempeñando en la constitución de la cultura del continente y sus relaciones con los países europeos, se ofrece al lector una excelente orientación en la bibliografía sobre el tema.

Aunque los textos presentados puedan parecer a primera vista heterogéneos, pueden identificarse dos claros ejes temáticos: en primer lugar, se proporciona la visión del papel de la traducción/intepretación en la configuración y emancipación de la(s) cultura(s) latinoamericana(s), en la que habría que incluir la importancia atribuida al papel social/cultural del traductor/intérpete (estudios de Adriana Domínguez Mares, Martha Pulido Correa, Lourdes Arencibia Rodríguez). Es segundo eje temático torna sobre los conceptos propios de la traducción latinoamericana como la «traducción cultural» (Ricardo Silva Santiestéban, Iván Pinto Román, Daniel Ricardo Yagolkovski) y el «canibalismo» como concepto innovador del área lusófona (Célia Luisa Andrade Pinto).

\section{Observaciones finales}

Las antologías de textos traductológicos representan, sin duda, un componente importante para la historia de la disciplina y acusan una característica específica, dado que sus elementos constitutivos pueden variar en el tiempo. En el ámbito checo-hispánico recobran una importancias especial: al ser compuestas de textos traducidos, ofrecen al público lector textos de difícil acceso en el original. Por otra parte, su edición, además del aporte que ofrecen para el conocimiento mutuo de dos concepciones culturales, parece reflejar otro aspecto que merece ser estudiado aparte, al menos en el ambiente checo: el de la evolución del hispanismo, que abre el paso al estudio de temas que hace unas décadas parecerían totalmente marginales, así como la reflexión del nivel alcanzado por los estudios de traducción e interpretación en el mundo hispanohablante, que despierta el interés de los especialistas a nivel internacional.

\footnotetext{
15 Original: Pulido Correa Martha (2012), Apuntes sobre la Translatio en los saberes curativos de América, in: Vega Cernuda Miguel Ángel (ed.) Traductores hispanos de la orden franciscana en Hispanoamérica, Lima, Universidad Ricardo Palma, p. 63-72

${ }^{16}$ Original: ARENCIBIA RodRíGUEZ Lourdes (1996-1997) La traducción en las tertulias literarias del siglo XIX en Cuba, Hieronymus Complutensis 4-5, p. 103-118

${ }^{17}$ Original: YAGOLKovski Daniel Ricardo (2011), Traducción del humor en literatura y en cine. in : Entre enigmas y certezas: cómo traducir literatura, Lima, Universidad de Ricardo Palma, p. 103-118

${ }^{18}$ Original: PInTo Román Iván (2011), Conciencia de la historia en el ejercicio de la traducción, in : Entre enigmas y certezas: cómo traducir literatura. Lima, Universidad de Ricardo Palma, p. 139-148 ${ }^{19}$ Orginal: ANDRADE PRADO Célia Luisa (2009) Muito além do cannibalismo: a teoria da traducção de Heraldo de Campos, in : Anais do X Encontro Nacionas de traductores \& IV Entcontro International de Traductores, Brasil, Editora da UFOP, p. 769-777
} 


\section{BIBLIOGRAFÍA:}

BolaÑos Sergio (2016), Introducción a la traductología. Autores, textos, cometarios. Bogotá, Universidad Nacional de Colombia.

HoDOUŠEK Eduard (1957), J. K. Tyl jako první český překladatel Calderóna, Časopis pro moderni filologii 39, p. 1-8.

KRÁLOVÁ Jana (2017), La recepción de la traductología latinoamericana en Chequia: inspiraciones temáticas, metodológicas y didácticas, Ars et humanitas 11 (2), p. 44-55.

KRÁlOVÁ Jana (2018), La traducción de la literatura española en la voz de sus protagonistas: Iberoamericana Pragensia XLVI (1), p. 97-104.

Králová Jana, Cuenca Drouhard, Miguel José (2013). Jiři Levý: una concepción (re)descubierta, Soria, Vertere.

LAMBERT José. (2010), The Languages of Translation. Keys to the Dynamics of Culture, in: MuÑoz-Calvo Micaela, Buesa GóMEz Carmen (ed.) Translation and Cultural Indentity: Selected Essays on Translation and Cross-cultural Communication, Newcastle, Cambridge Scholars Publishing, p. 33-60.

LEVÝ Jiří (1957 [1996]), České theorie prekladu, Praha, Státní nakladatelství krásné literatury, hudby a umění.

LEVÝ Jiř́ (1963), Uměni prekladu. Praha. Československý spisovatel.

LEVÝ Jiří (1971), Bude literárni věda exaktní vědou? Praha, Československý spisovatel.

MraČKOVÁ VAVROUŠOVÁ, Petra. (2018), Soudobá recepce díla Jiř́ho Levého ve Španělsku a Iberoamerice, AUC Philologica 2, p. 143-153.

RUBÁŠ Stanislav (2011), Slovo za slovem, Praha, Academia.

SABIO PINILlA José Antonio (2011), ¿Es la antología un género? A propósito de las antologías sobre la traducción, Hikma 10, p. 159-174.

SABIO PINILla José Antonio (2010), Las antologías sobre la traducción en España y Portugal: revisión crítica, Sendebar 21, p. 7-20.

SANTOYo Mediavilla Julio César (2011), Iberian translation history: what we know and do not know, in: KITTEL Harald (ed.), Übersetzung, Translation, Traduction, Berlin, Boston, Walter de Gruyter, p. 1982-1984.

SERRANO BERTOS Elena (2014) Jiř́ Levý: una concepciónn (re)descubierta, Linguistica Pragensia 1, p. 84-87.

SERUYA Teresa, LIEVEN D'HULST Alexandra, ASSIS Rosa, LIN MONIZ Maria

(2013), Translation in Anthologies and Collections (19th and 20th Centuries). Amsterdam: John Benjamins (Benjamins Translation Library, 107).

ULIČNÝ Miloslav (2016), Hispánská literatura v Českych teoriich překladu Jiř́iho Levého, Svět literatury 54, p. 165-173

VAVROUŠOVÁ Petra (2013), Sedm tváŕi translatologie, Praha, Karolinum.

VAVROUŠOVÁ Petra (2015), Překlad jako most mezi kulturami, Praha, Karolinum.

VAVROUŠOVÁ Petra (2015a), Reseña: Jiří Levý: una concepción (re)descubierta, Meta 3, p. 644-645.

VAVROUŠOVÁ Petra (2016), Al margen de la recepción recíproca de las teorías de la traducción checa e hispana, in: WILK-RACIĘSKA Joanna, DEDITIUS Sabina, NoWAKOWSKA-GŁUSZAK Anna (ed.) Relecturas y nuevos horizontes en los 
estudios hispánicos: cultura y traduccion, Katowice: Wydawnictwo Uniwersytetu Śląskoegi, p. 136-146.

VEGA CERNUDA Miguel Ángel (1994), Textos clásicos de la teoría de la traducción, Madrid, Cátedra.

Vega Cernuda Miguel Ángel (2011), El caso Levý: Fenomenología de su recepción y valoración de sus aportanciones en el contexto de la traductología de la época, in: KrÁlovÁ Jana (ed.), Posibilidades y límites de la comunicación intercultural, Praha, Karolinum. p. 11-20.

VEGA CERnUdA Miguel Ángel (2013), Prólogo. Levý, un pensador minorizado en la traducción, in: KRÁLOVÁ Jana, CUENCA DROUHARDT Miguel (ed.), Jiř́ Levý: una concepción (re)descubierta, Soria, Vertere, p. 13-18. 